Pier Giorgio Masci Steven Dymarkowski Jan Bogaert

\section{Valvular heart disease: what does cardiovascular MRI add?}

Received: 22 March 2007

Revised: 26 June 2007

Accepted: 12 July 2007

Published online: 29 August 2007

(C) Springer-Verlag 2007

Electronic supplementary material The online version of this article (doi:10.1007/ s00330-007-0731-x) contains supplementary material, which is available to authorized users.

P. G. Masci · S. Dymarkowski .

J. Bogaert $(\bowtie)$

Department of Radiology, Gasthuisberg

University Hospital,

49 Herestraat,

Leuven, 3000, Belgium

e-mail: jan.bogaert@uz.kuleuven.ac.be

Tel.: +32-16-343782

Fax: +32-16-343765
Abstract Although ischemic heart disease remains the leading cause of cardiac-related morbidity and mortality in the industrialized countries, a growing number of mainly elderly patients will experience a problem of valvular heart disease (VHD), often requiring surgical intervention at some stage. Doppler-echocardiography is the most popular imaging modality used in the evaluation of this disease entity. It encompasses, however, some non-negligible constraints which may hamper the quality and thus the interpretation of the exam. Cardiac catheterization has been considered for a long time the reference technique in this field, however, this technique is invasive and considered far from optimal. Cardiovascular magnetic resonance imaging (MRI) is already considered an established diagnostic method for studying ventricular dimensions, function and mass. With improvement of MRI soft- and hardware, the assessment of cardiac valve function has also turned out to be fast, accurate and reproducible. This review focuses on the usefulness of MRI in the diagnosis and management of VHD, pointing out its added value in comparison with more conventional diagnostic means.

Keywords Magnetic resonance imaging - Valvular heart disease · Valvular regurgitation - Valvular stenosis

\section{Introduction}

A number of imaging modalities are currently available to evaluate valvular heart disease (VHD) in a comprehensive manner allowing correct assessment of both valve morphology and function. Doppler-echocardiography is the most frequently used tool for this purpose because it is cost-effective, widely available and, in the majority of the cases, provides sufficient information for clinical patient management and possible surgical planning [1]. For a long time, cardiac catheterization and invasive angiography have been regarded as the "gold standard". However this invasive approach exposes patients to radiation and iodinated contrast media, carrying non-negligible risk of life-threatening complications but nonetheless far from optimal [2], especially regarding the precise quantification of valvular regurgitation. Due to considerable improvements in hard- and software design in the last decade, magnetic resonance imaging (MRI) has claimed its role as a central player in a large variety of cardiac diseases, not infrequently offering unique information about tissue characterization, disease severity and impact on cardiac function and perfusion. Because of both its high accuracy and reproducibility, MRI has become the preferred imaging modality in an increasing number of clinical trials. Also in the field of VHD, considerable progress has been achieved [3]. Recently, multidetector-row computed tomography (MDCT) has also been proposed as a new diagnostic tool in the evaluation of cardiac valves. Although this technique yields excellent spatial resolution, being able to clearly depict cardiac valve anatomy, its limited temporal resolution and the need to use iodinated contrast media and ionizing radiation will limit its applicability in daily routine $[4,5]$. Moreover, the lack of functional information about the valve disease severity will not favor the use of this imaging modality. 
In this review, we discuss how cardiovascular MRI techniques can be applied to study VHD patients, in particular highlighting the additional value of this imaging modality over other conventional diagnostic means.

\section{VHD: what kind of MRI sequences do we need?}

Before we can answer this question, we have to address several crucial issues required for the diagnosis and management of patients with or presumed to have VHD (see Table 1). The first topic concerns valve morphology, the second valvular function and the third how valve abnormalities influence cardiac function. Often additional information is needed, such as the exclusion of associated coronary artery disease in patients planned for elective valve replacement or the ruling out of thrombi in the atria/ appendages (e.g., patients with mitral stenosis). In patients with endocarditis in whom an infectious pseudoaneurysm is suspected, the MRI exam needs to be focused on the area surrounding the affected valve. In other cases, for instance in bicuspid aortic valve patients, an additional study of the thoracic aorta may be required to exclude aorta abnormalities (e.g., aneurysm and coarctation).

Table 1 Essential information in the diagnosis and management of VHD patients

\begin{tabular}{l} 
Vital data on the diagnosis and management of VHD patients \\
\hline 1. Valve morphology \\
number of leaflets \\
integrity of leaflets (e.g., rupture, fusion, prolapse) \\
integrity of tendinous chords \\
pathologic features (e.g., calcification, vegetations) \\
perivalvular morphology (e.g., abscess, pseudoaneurysm) \\
2. Valve function \\
opening pattern (e.g., bicuspid, tricuspid) \\
coaptation pattern (e.g., normal, mal- or noncoaptation) \\
valve orifice/valve circumference \\
mean/peak systolic flow + calculation of transvalvular gradient \\
regurgitant flow/regurgitant fraction \\
3. Ventricular function \\
volumes \\
systolic/diastolic function \\
wall mass \\
regional wall motion \\
4. Additional information \\
great vessels [diameter/associated pathology (e.g., coarctation)] \\
thrombi \\
coronary artery disease \\
myocardial infarction/scarring \\
\hline
\end{tabular}

Currently, spin-echo double inversion-recovery MRI techniques (e.g., fast or turbo spin-echo technique) are usually not the first choice to examine VHD patients, although sometimes they still may offer valuable information (e.g., in patients with endocarditis-related pseudoaneurysm). These dark-blood sequences are rarely able to depict cardiac valves in a reliable manner, mostly when pathological processes alter the tissue valve composition and morphology. For instance, calcified or fibrotic valves have very low signal intensity and are therefore difficult to discriminate from the surrounding (dark) blood [6]. Most of the morphologic as well functional information is currently obtained by using cine MRI sequences. A large series of images (usually in the order of 20-40) are obtained over the length of one cardiac cycle in a plane through the heart. These can be played in a cine mode, offering dynamic information. From a practical point of view, cine-MRI sequences are usually acquired within a single breath-hold, although it is currently possible to perform these studies in real-time fashion, which, however, reduces spatial and temporal resolution. From a sequence point of view, the spoiled gradient-echo sequences have been replaced by the newer balanced steady-state freeprecession (SSFP) sequence, offering a much higher intrinsic contrast between blood and the surrounding cardiac structures. Also, visualization of thin structures like valve leaflets and assessment of valve area have benefited substantially by using this new type of brightblood sequence [7]. From an imaging point of view, usually a combination of longitudinal and perpendicular imaging planes through the valve of interest are selected.

These bright-blood sequences do not only show valve leaflet motion and function, eventually demonstrating abnormal leaflet coaptation during valve closure (e.g., valve insufficiency) or alteration of physiological leaflet excursion during valve opening (e.g., valve stenosis), but they also depict alteration of the normal blood flow pattern. Turbulent flow pattern across diseased valves, due to flow acceleration or loss of flow homogeneity, is shown as an area of low signal intensity (signal void) [8]. Although disease severity is often visually graded according with the extent of signal loss, one should bear in mind that the degree of the signal loss depends on both sequence design and imaging parameters (mainly echo time) [9, 10]. Fortunately, the new balanced-SSFP sequences are relatively flow-insensitive [11] and show good sensitivity and diagnostic accuracy for visual identification of valvular dysfunction and semi-quantitative estimation compared with the older spoiled gradient-echo sequences and other conventional diagnostic techniques (i.e., Doppler-echocardiography and cardiac catheterization) [3]. Parallel imaging is nowadays routinely used in conjunction with balanced-SSFP cine MRI, so that breath-hold length can be substantially reduced [12].

Phase-contrast or velocity-encoded cine MRI is a highly attractive technique to visualize and to quantify flow, enabling to calculate blood flow velocities and volumes, 
such as ventricular stroke volumes, cardiac output, and regurgitation flow in insufficient valves or high-velocity jet through stenotic valves [13]. Hydrogen protons moving along a magnetic field gradient acquire a phase shift relative to stationary spins. For linear field gradient, the entity of this phase shift is directly proportional to the velocity of moving protons. Because phase shifts can arise from other factors (e.g., field inhomogeneity), a second velocity-compensated phase image is acquired and subtraction yields the actual phase relationship of the protons [14]. The phase-shift difference between stationary and moving magnetic moments is used for voxel-by-voxel calculation of velocities. Measurement of the mean velocity for all pixels in a region of interest of known area enables the calculation of the instantaneous flow volume at any point in the cardiac cycle. Calculation of the flow volume per heart beat can be made by integrating the instantaneous flow volumes for all frames throughout the cardiac cycle. This technique has extensively been validated in vitro and in-vivo, and is extremely accurate and reproducible [15]. The information obtainable from phase-contrast MRI generates magnitude and phase imaging (Fig. 1). The former is used as anatomical reference and is a bright-blood gradient-echo image, whilst on the phase image each voxel velocity is shown on a gray scale. The thoracic wall, which is not moving during sequence acquisition, is used as a reference for zero velocity, while moving voxels in blood vessels appear black or bright, depending on their flow direction. Their intensity is directly dependent on their flow velocity. Velocity encoding can be applied in any direction (throughplane, left to right, up and down), though for flow quantification through-plane imaging is required.

There are several technical issues that must be addressed when performing phase-contrast velocity mapping. Firstly, for phase-contrast image quality it is particularly relevant to set the flow-sensitizing gradient (encoding velocity) at level as close as possible to the expected peak velocity. This is paramount since too low encoding velocity induces aliasing phenomenon, whereas by setting a too high value decreases the relative flow amplitudes, hampering velocity and flow data interpretation [15]. Secondly, phase-contrast imaging should be performed as close as possible to the center of the main magnetic field $\left(\mathrm{B}_{0}\right)$. This ensures phase errors introduced by eddy currents and Maxwell terms are kept at minimum level. Thirdly, the imaging plane should be perpendicular to the vessel. This ensures that the velocity vectors of the majority of the voxels are perpendicular to the imaging plane. This can be achieved by planning the through-plane imaging position from two perpendicular views. Normally the plane misalignment generates an underestimated measure of the true in-plane velocity; however, this error is small (e.g., $6 \%$ at $20^{\circ}$ misalignment) [16]. Fourthly, the imaging plane should not be at the level of the valve but just proximal or distal to the valve annulus. This lessens the artifacts secondary to eddy currents and the motion of the valve annulus. One possible method to

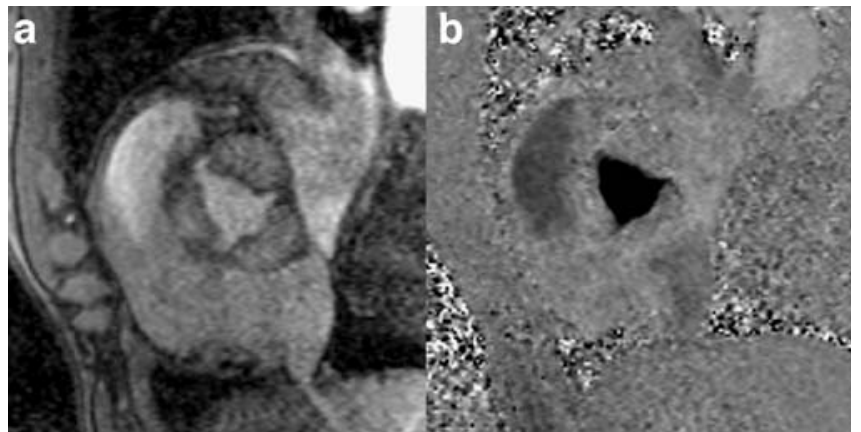

c

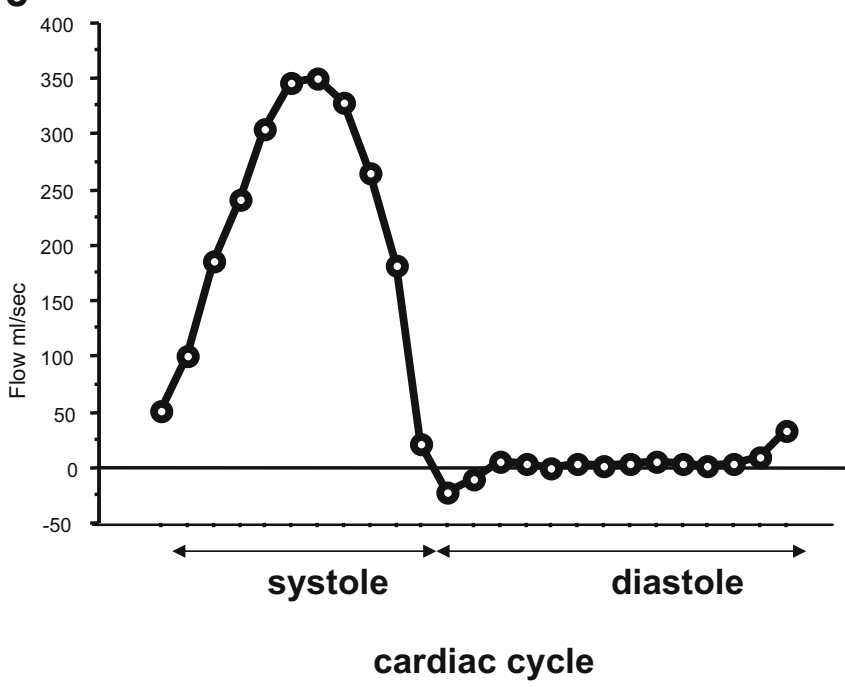

Fig. 1 Phase-contrast MRI of an aortic valve in a normal volunteer. Magnitude image (a), phase image (b), corresponding flow map (c). Images a and $\mathbf{b}$ show peak of systolic ejection: three-leaflets valve and presence of normal orifice are well visible on magnitude image (a). Phase image (b) shows forward blood flow (shown as a dark area) through opened aortic valve. Stationary tissues are shown as gray. Flow map (c) obtained by delineating the aortic valve on all cardiac time frames, shows a peak systolic forward flow $(77 \mathrm{ml} /$ heart beat) and absent significant reversal diastolic flow

compensate the through plane valve motion is to use a moving slice velocity mapping technique [17]. This experimental method enables the imaging slice to follow the valve annulus during the cardiac cycle, reducing velocity offsets. However, this technique is not yet currently available in MRI scanners.

When scanning VHD patients, imaging is started with scout images to localize the heart and to determine the subsequent image planes. Next, the diseased valve is studied with bright-blood cine MRI technique by using usually a series of different, often perpendicular, imaging planes. Subsequently, phase contrast MRI is applied to quantify the severity of stenosis and/or regurgitation. In patients with multi-VHD this scheme is applied not only to the diseased but also to nondiseased valves in order to accurately quantify the valvar dysfunction. It is also 
mandatory to assess the impact of VHD on ventricular volumes, mass, and function for which cardiac MRI is the gold standard [18, 19]. In patients with diminished left ventricular function or with suspected concomitant coronary artery disease additional stress testing, by using either incremental doses of dobutamine (stress function) or a vasodilator agent (stress perfusion), may be required. An alternative is adding MR coronary angiography, which enables to depict or rule out coronary artery stenoses in the proximal coronary artery segments with a moderately high accuracy [20]. Late or delayed contrast-enhanced MRI, using an inversion-recovery gradient-echo sequence, allows excluding concomitant myocardial infarction/scarring and this is, moreover, a sensitive technique to detect intracavitary thrombi [21]. In summary, several series of MRI techniques are currently available, allowing a fully comprehensive evaluation of the diseased valve(s), the impact on cardiac function and to rule out VHD complications (e.g., intracavitary thrombi, aneurysm formation). Each MRI protocol should be fine-tuned according to the clinical problem.

\section{Valvular regurgitation}

Accurate quantification of valvular regurgitation is important but remains a challenging issue. Doppler-echocardiography is the imaging technique of choice. However, several considerations need to be emphasized. First of all, transthoracic echocardiography (TTE) has a good accuracy in detecting dysfunction of the left-heart valves, whereas its accuracy significantly decreases for right-heart valves [22]. On parasternal views, the right-heart, and in particular the pulmonary valve, is often poorly visible, and TEE is often not of help. Moreover, TEE is a semi-invasive procedure often requiring sedation. Another major limitation of echocardiography in assessing valvular regurgitation severity is the lack of true quantification parameters. In daily clinical practice, color-Doppler imaging is often used. The color jet area, however, depends on several factors other than regurgitation severity, such as pressure gradient across the valve, compliance of the receiving chamber, the eccentricity of jet and instrumentation parameters [23]. More recently, the effective regurgitation orifice area (EROA) has been described as a useful quantitative measure in mitral regurgitation [24]. This parameter, derived from the proximal isovelocity surface area (PISA) method, however is difficult to apply in case of eccentric jet or when the image quality does not allow an optimal individuation of convergence flow [25]. Therefore, although the PISA method holds promise, it is still far from routine clinical application. Cardiac catheterization can provide some hemodynamic information, but encompasses several drawbacks. Besides being invasive, the assessment of regurgitation severity is subjectively based on one or two projections. Moreover, occurrence of ectopic beats during ventriculography may further hamper assessment of atrioventricular valve regurgitation, and similarly, the severity of aortic regurgitation may be overestimated if during aortography the pigtail catheter impedes the leaflet coaptation.

Valvular regurgitation is visible on cine MRI as an area of signal loss extending from the dysfunctional valve into the proximal chamber [9] (Fig. 2). The signal loss can be graded in a similar way to X-ray angiography: grade $1=$ signal loss close to the valve; grade $2=$ signal loss extending into the proximal chamber; grade $3=$ signal loss filling the whole of the proximal chamber; grade $4=$ signal loss in the receiving chamber throughout the relevant half of the cardiac cycle [26] (Fig. 3). This semi-quantitative
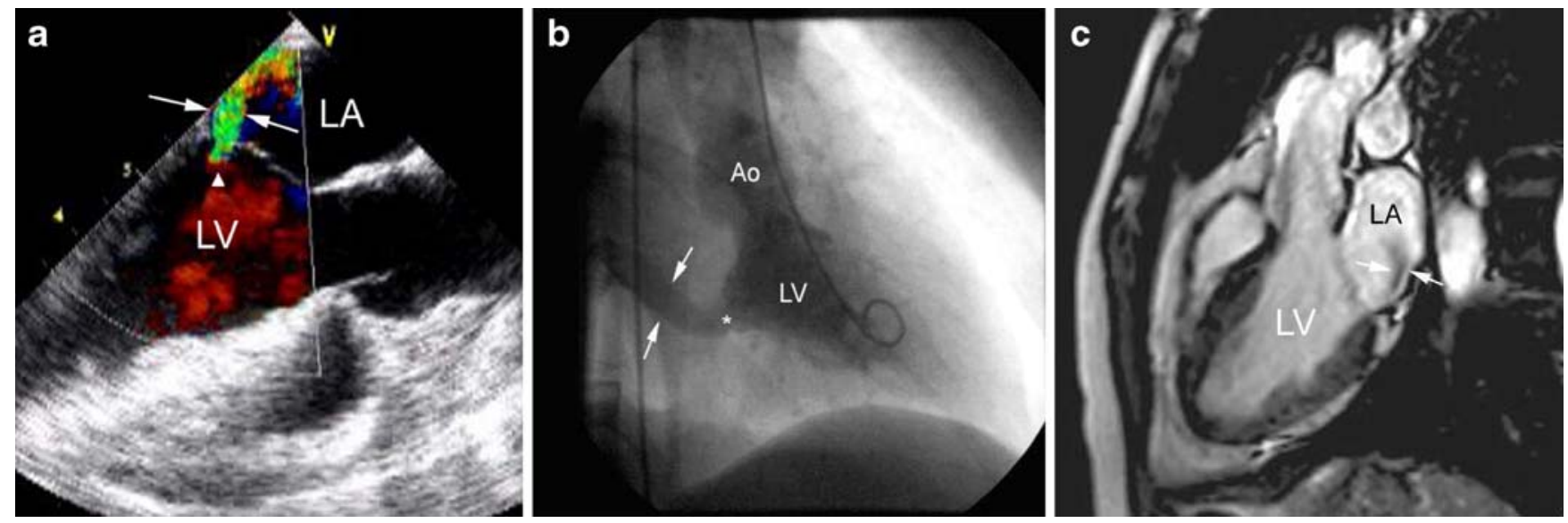

Fig. 2 Eccentric mitral regurgitation. Transesophageal echocardiography (a) shows eccentric jet (arrows) directed from left ventricle $(L V)$ into left atrium $(L A)$ (movie $2 \mathrm{~A})$. The jet impinges on LA wall ("wall jet") dissipating part of its kinetic energy ("jet momentum"), resulting in underestimation of mitral regurgitation severity. Contrast ventriculography (b), obtained in right anterior oblique view,

confirms eccentric regurgitant jet $(*)$ (arrows) (movie 2B). Balanced SSFP cine-MRI in LV inflow-outflow view (c) clearly shows eccentric mitral regurgitation (arrows) (movie 2C). Valve defect was not seen on vertical long and horizontal long axis views (not shown), emphasizing the importance of multiplane scanning in eccentric regurgitation 
Fig. 3a-d Severe aortic regurgitation. Bicuspid aortic valve (latero-lateral rim) with rupture of anterior leaflet and severe aortic regurgitation with left ventricular (LV) volume overload. Cine MRI (SSFP sequence) perpendicularly oriented through aortic valve (a), along LV outflow tract (b). Magnitude (c) and phasecontrast (d) MR image perpendicular through LV outflow tract. In a, closure of bicuspid aortic valve (arrowhead) but presence of area of signal void (arrow) in middle of anterior leaflet corresponding to the area of rupture. In $\mathbf{b}$, anterior leaflet rupture leads to complex and important holodiastolic aortic regurgitation (movie 3B). Severity of aortic insufficiency is quantified using phase-contrast imaging (movie $3 \mathrm{C}$ ), where regurgitation is depicted as a pinpoint dark area (c, d; arrows). Following parameters are derived: regurgitation flow volume: $77 \mathrm{ml}$; regurgitation fraction: $46 \%$. Chronic volume overload has led to important eccentric LV hypertrophy (end-diastolic volume: $328 \mathrm{ml}$; ejection fraction: $58 \%$; mass: $195 \mathrm{~g}$ )

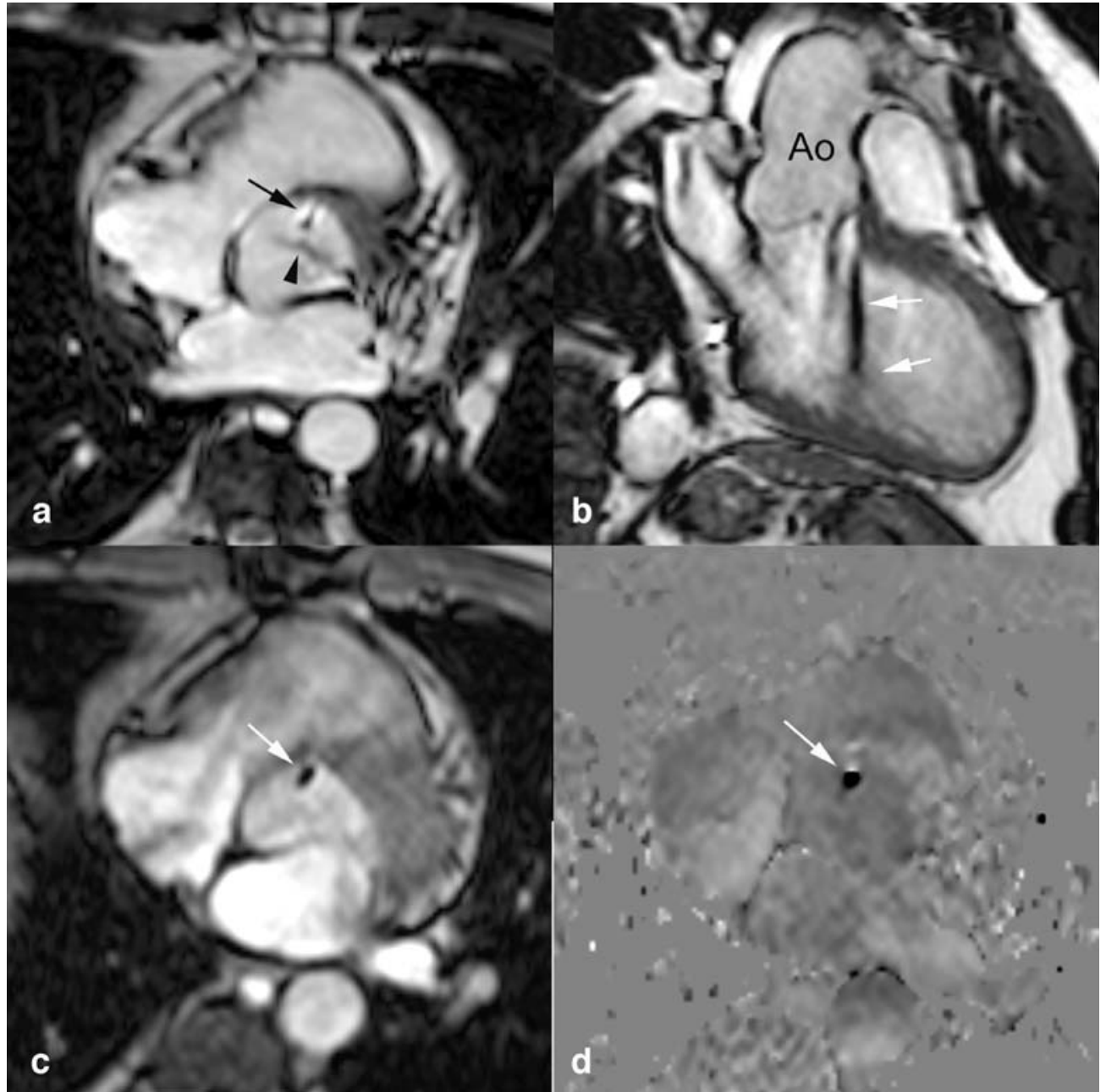

method has been validated, although its reproducibility is quite unsatisfactory [27] and it is affected by the same intrinsic limitations as color-Doppler echocardiography and invasive angiography. A combination of ventricular volumetric quantification and phase-contrast MRI, however, allows accurate and reproducible assessment of valvular regurgitation. In normal conditions, according to the Frank-Starling principle, the stroke volumes from both ventricles are equal. In the presence of single incompetent valve, the difference in stroke volumes between the ventricles yields the regurgitation volume [28]. This method is, however, not applicable in case of multivalvular regurgitation, or if a cardiac shunt coexists. Phase-contrast MRI enables direct quantification of the regurgitation flow and fraction. In our experience, using a perpendicularly oriented imaging plane just below the diseased valve is the most appropriate approach to quantify valve regurgitation. The backward flow is shown on the phase image as an area of increased or decreased signal intensity during cardiac cycle when the diseased valve is expected to be closed (e.g., diastolic backward flow for aortic regurgitation). Delineation of the area of interest during on all image frames, yields the forward and regurgitation flow. Dividing the backward flow by the forward flow, the regurgitation fraction is derived [29, 30] (Figs. 4, 5). For the atrioventricular valves, an indirect method may be preferable because often it yields more accurate and consistent results than the direct approach. This indirect method consists of subtracting the forward flow of the great vessel (e.g., aortic forward flow, for the quantification of mitral valve regurgitation), calculated by phase-contrast MRI, from the stroke volume (volumetric computation) of the corresponding ventricle (e.g., left ventricle for mitral valve regurgitation). Normalizing the regurgitation volume to the ventricular stroke volume, the regurgitation fraction is calculated. Use of regurgitation fraction should be recommended as this parameter has the advantage of being relatively insensitive to other concomitant valve abnormalities [28]. Recently, the regurgitation fraction limits for mitral and aortic regurgitations have been estimated by using cardiac MRI: mild $\leq 15 \%$; moderate $16-25 \%$; moderate-severe $26-48 \%$; severe $>48 \%$ [30]. In patients with multivalvular disease, it is advisable to use a combination of volumetric ventricular quantification and phase-contrast MRI of all cardiac valves. 
Fig. 4 Functional mitral regurgitation in a 44-year-old woman with idiopathic dilated cardiomyopathy. Functional mitral regurgitation is caused by dilation of mitral ring (loss of sphincterlike function) and tethering of both leaflets due to papillary muscles displacement. Cine MRI (SSFP sequence) in horizontal long-axis (a) and vertical long-axis (b), both at systole. Regurgitant flow appears as stripe-like jet (arrows) into left atrium. Phase-contrast MRI (see movie 4) obtained perpendicularly through the mitral valve. During systole (c), regurgitant flow appears as a pinpoint white spot (black arrows), whereas during diastole (d) the inflow through mitral valve is shown as an oval black structure (white arrows). A regurgitation fraction of $12 \%$ was derived

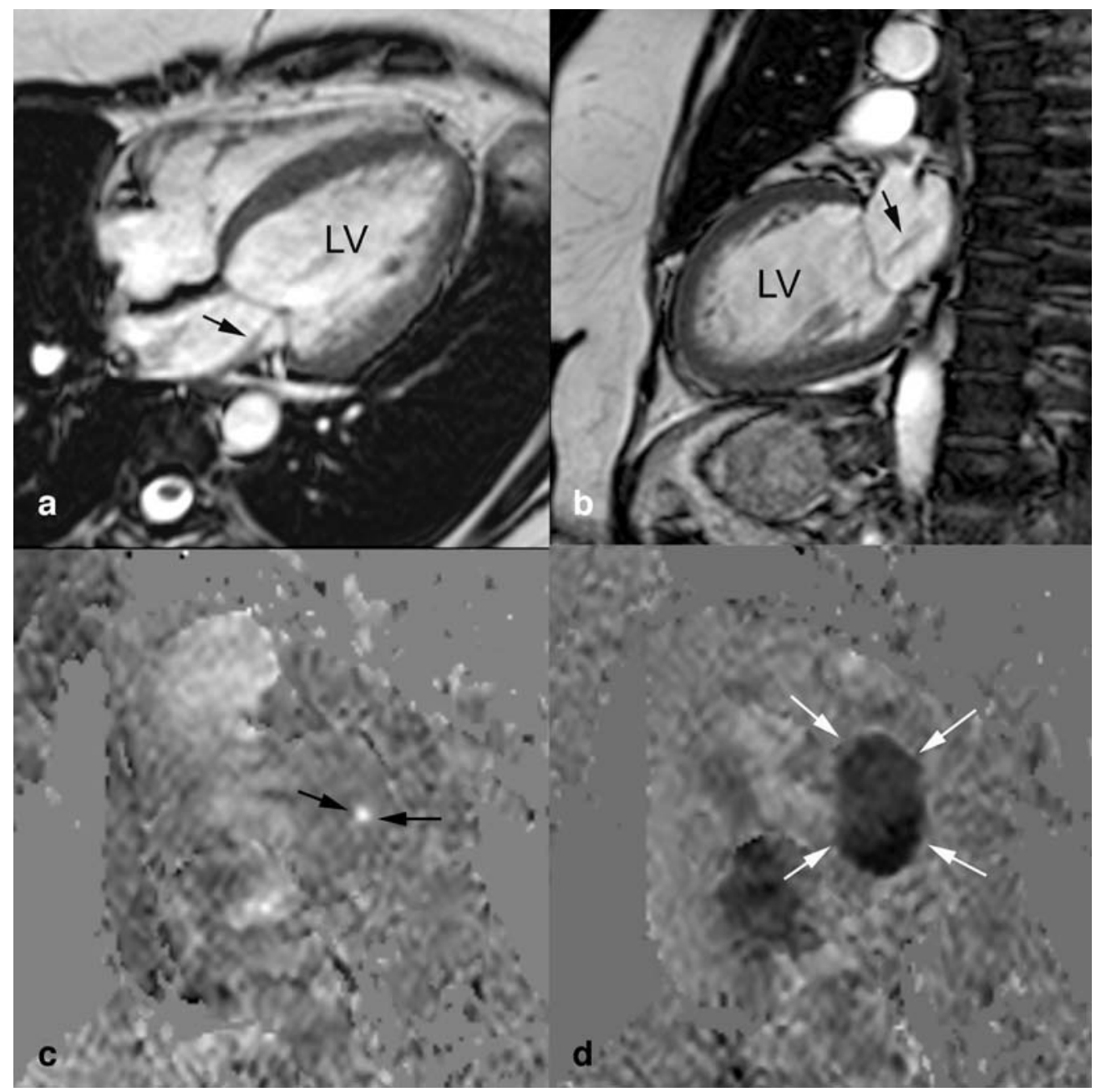

Cardiac MRI is widely recognized as the gold standard for quantification of ventricular volumes and systolic function being superior to two-dimensional (2D) echocardiography in assessing normal, dilated and hypertrophied hearts $[18,19,31]$. This is of vital importance in planning the correct time for cardiac surgery. In fact, in valvular regurgitation the clinical status is misleading as symptoms appear only in the advanced phase when irreversible ventricular damage might have already occurred. Therefore, an accurate assessment of ventricular volumes and systolic function and their follow-up over time represents the most useful tool for timing of surgical intervention [32].

Cardiac MRI has an indisputable leading role in the assessment, quantification and follow-up of patients affected by pulmonary regurgitation. This condition is very rare in isolated form, but it is extremely common in patients after surgical repair for tetralogy of Fallot. Most of these patients have a good overall prognosis [33]; however, in cases with severe pulmonary regurgitation, right ventricular dysfunction may supervene, being responsible for late mortality and morbidity. In this setting, it is crucial to correctly quantify the pulmonary insufficiency and to precisely assess the right ventricular volumes and function. Indeed, a delayed surgical intervention may result in unfavorable prognosis whereas an undue or precocious operation must be avoided. As mentioned previously, Doppler-echocardiography encompasses important limitations in evaluating right ventricular function and quantifying pulmonary regurgitation [22]. In contrast, MRI enables accurate quantification of right ventricular volumes, mass and function and also the correct assessment of pulmonary regurgitation (Fig. 6). A regurgitation fraction $\geq 40 \%$ is considered severe [34]. This cut-off value is lower than that identified for mitral and aortic valves because in the pulmonary arterial system, in contrast to the systemic arterial tree, there is no reversal flow during early diastolic phase. This phenomenon is due to high compliance of the pulmonary tree, which behaves as a valve.

\section{Valvular stenosis}

Doppler-echocardiography has an established role in the diagnosis and quantification of valvular stenosis [35]. In this 
Fig. 5 Aortic regurgitation due to annulo-aortic ectasia. A 52year-old man affected by annulo-aortic ectasia (ascending aorta diameter: $50 \mathrm{~mm}$ ). Four frames of cine MRI (SSFP sequence) of left ventricular inflow-outflow view: end-diastole (a), mid systole (b), early diastole (c) and mid diastole (d). Stretching of normal aortic valve cusps (not shown) because of aortic root dilatation with consequent loss of coaptation resulting in eccentric valve regurgitation. Regurgitant jet is sharply defined in its proximal portion, where it abuts the anterior mitral leaflet, and then fans out (arrows) into the LV (d; movie 5). Regurgitation fraction is $30 \%$ with LV overload (enddiastolic volume: $257 \mathrm{ml}$; ejection fraction: $52 \%$ )

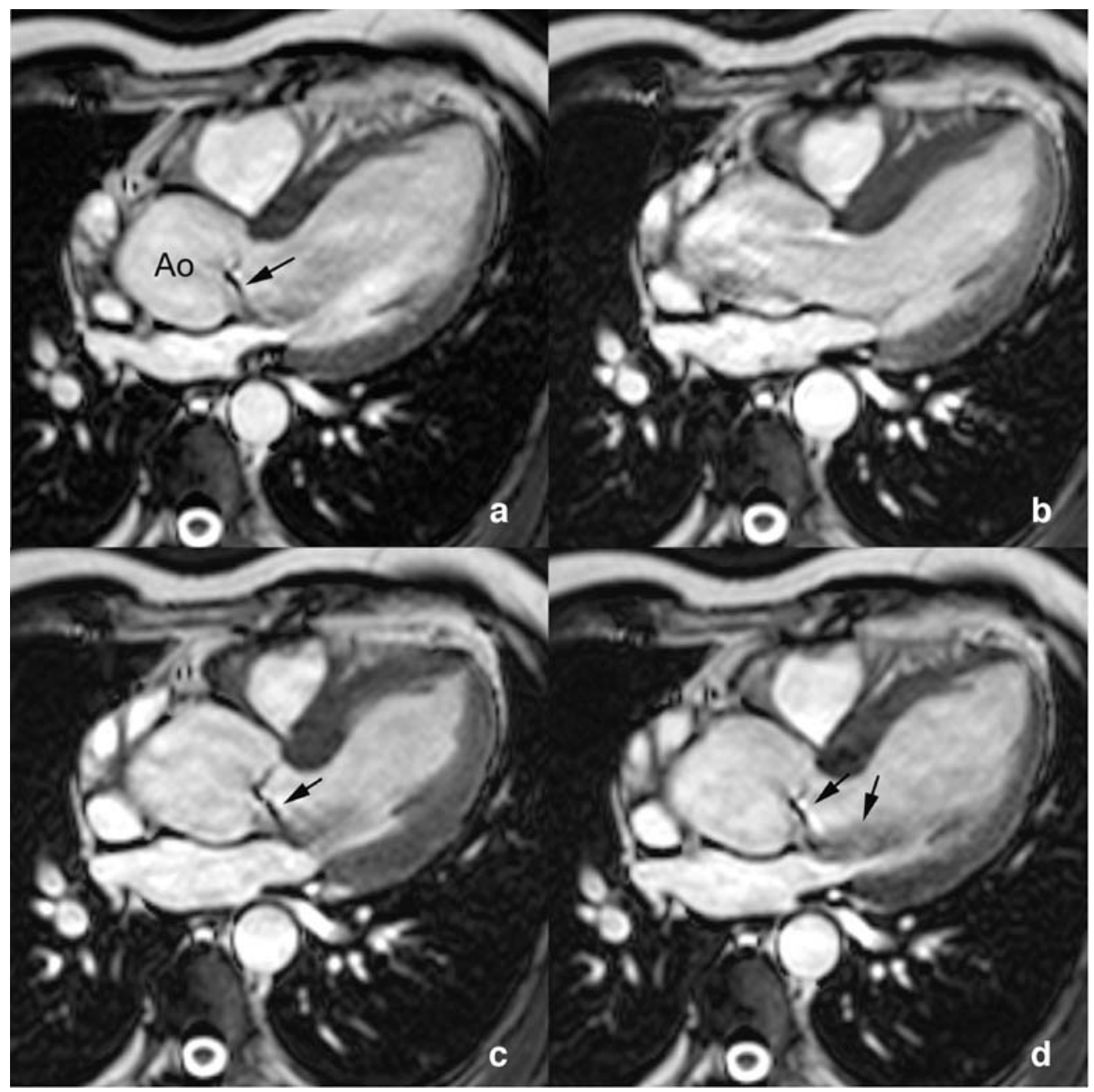

scenario, clinical symptoms and valve area estimation represent the mainstay for patient management. The valve area can be measured by using the continuity equation or the planimetric method. Both of them have been largely used in echocardiography and have been translated into cardiovascular MRI. The continuity equation relies on the principle of conservation of mass and its formula is mainly used to evaluate the functional aortic valve area. By measuring the velocities in the left ventricle outflow tract $\left(\mathrm{LVOT}_{\mathrm{vel}}\right)$ and ascending aorta $\left(\mathrm{Ao}_{\mathrm{vel}}\right)$, and by calculating the LVOT area, the aortic valve area $\left(\mathrm{Ao}_{\text {area }}\right)$ can be derived [36]:

$$
\mathrm{Ao}_{\text {area }}=\left(\mathrm{LVOT}_{\text {area }}\right) *\left(\mathrm{LVOT}_{\text {vel }}\right) /\left(\mathrm{Ao}_{\text {vel }}\right) \text {. }
$$

Cine MRI can correctly identify the presence of valvular stenosis by signal loss, originating in the diseased valve and extending into either the receiving chamber or the great vessel. Detection and semiquantitative assessment of valve dysfunction are useful because they represent the first step for further quantitative analysis. By using phase-contrast MRI, it is possible to perform through-plane (velocity jet perpen- dicular to the imaging plane) or in-plane (velocity jet parallel to the imaging plane) velocity mapping. The in-plane method allows visualizing the entire jet and the points within the jet peak velocity area are easily identified. However, not all jets are straightforwardly aligned in a single 2D plane and, for tight narrow jets, there may be partial volume averaging and motion within the imaging slice, and the peak velocity may not be accurately depicted. Therefore, the through-plane is preferred in order to accomplish reliable velocity and flow measurements across the valve. It is important to choose correct position of the plane, which must be orthogonal to jet flow. This can be achieved by planning the through-plane measurement by using two perpendicular reference images visualizing the stenotic valve. Once the peak velocity has been obtained, the gradient across the valve can be estimated by the modified Bernoulli equation [37]:

$\Delta P=4 V^{2}$

[where $\Delta P=$ the pressure drop across the stenosis ( $\mathrm{mmHg}$ ) and $V=$ peak velocity $(\mathrm{m} / \mathrm{s})]$. 
Fig. 6 Severe pulmonary regurgitation post tetralogy of Fallot repair in a 20-year-old man. SSFP cine MRI (a) of the right ventricle (RV), RV outflow tract and main pulmonary artery (PA) obtained at diastole. Abnormal coaptation of pulmonary cusps (arrowheads) results in severe pulmonary regurgitation (arrows). Phase image perpendicular to RV outflow tract during systole (b) indicates forward blood flow ("bright" appearance) across outflow tract during systole (arrows). Corresponding phase image during diastole (c) shows severe backward flow ("black" appearance), resulting in regurgitation fraction of $46 \%$, and reduced RV ejection fraction of $43 \%$ (d forward flow is shown in green, severe regurgitant diastolic flow in red)

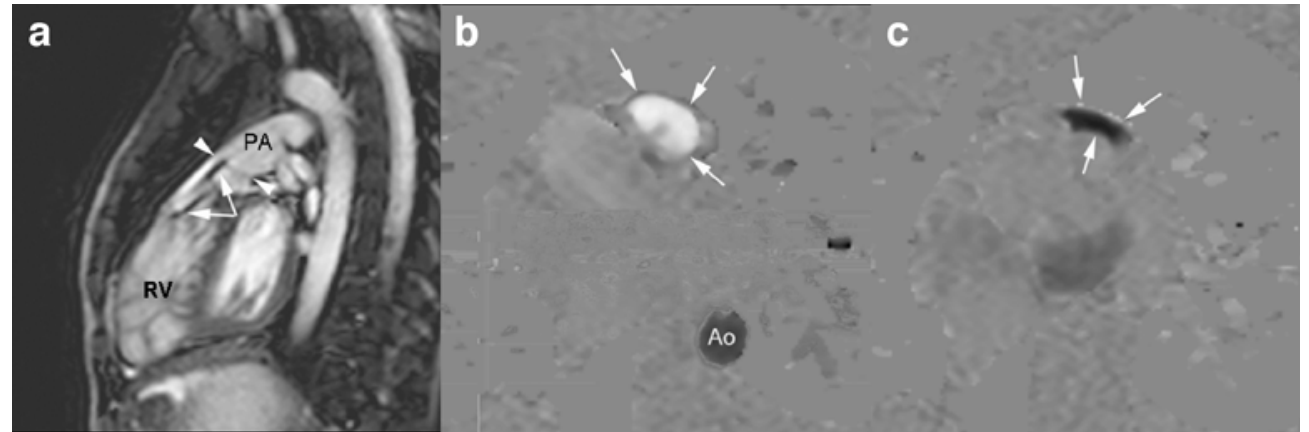

d

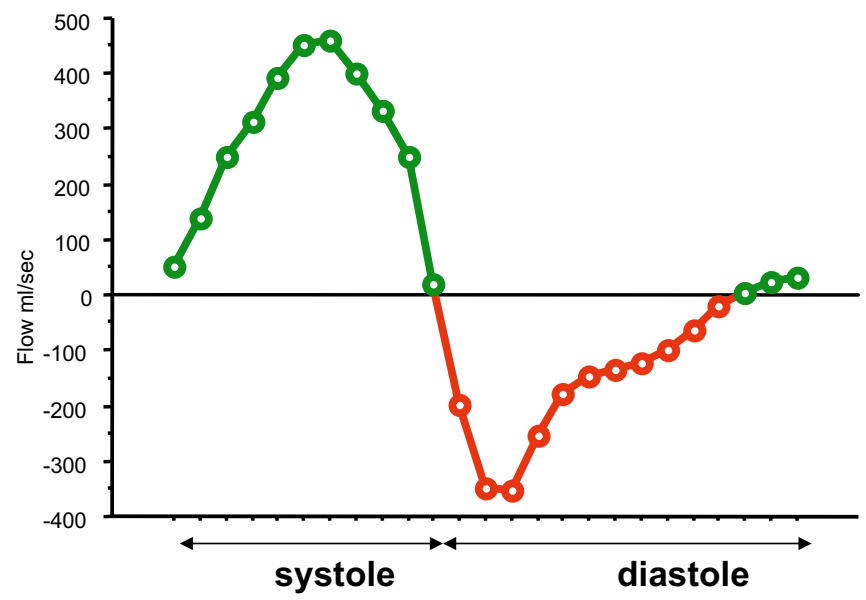

cardiac cycle
The main advantage of cardiac MRI over echocardiography is the easy alignment of the imaging plane in any direction without any limitation due to acoustic window. As previously described, the velocity encode gradient must be correctly chosen in order to have good sensitivity and accuracy of measurements (Fig. 7). Most scanners now have fast phase-contrast velocity mapping sequences that can be acquired in a single breath-hold. This enables an estimate of the peak velocity to be made before progressing to the more time-consuming conventional phase-contrast velocity mapping sequence.

Aortic valve stenosis is the most common valve disease resulting in valve replacement [38]. Correct assessment of stenosis severity is necessary before valve replacement is

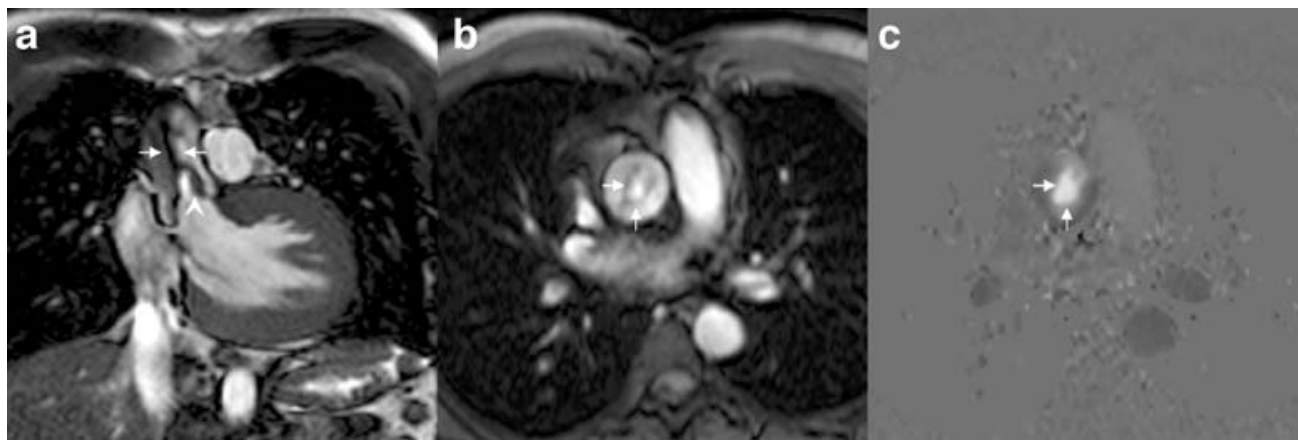

Fig. 7 Bicuspid aortic valve in a 40-year-old man with prevalent stenosis. Bicuspid aortic valve, type antero-posterior rim, is well seen on magnitude (b) and phase images (c) (arrows). Valve abnormality results in stenosis (peak velocity $4.2 \mathrm{~m} / \mathrm{s}$; peak gradient: $70 \mathrm{mmHg}$, and planimetry aortic orifice: $1.1 \mathrm{~cm}^{2}$ ), although moderate aortic regurgitation is also present (not shown; regurgitation fraction of $30 \%$ ). Aortic valve abnormalities have led to severe left ventricular remodeling (end-diastolic volume: $400 \mathrm{ml}$; ejection fraction 46.5\%). Balanced-SSFP cine-MRI (a) during systolic ejection phase shows the "doming sign" (arrowhead) of left cusp and signal void in ascending aorta due to turbulent blood flow (arrows). Cusp doming is very often detected in congenital aortic valve stenosis as valve leaflet mobility is partially preserved due to lack of extensive calcification 


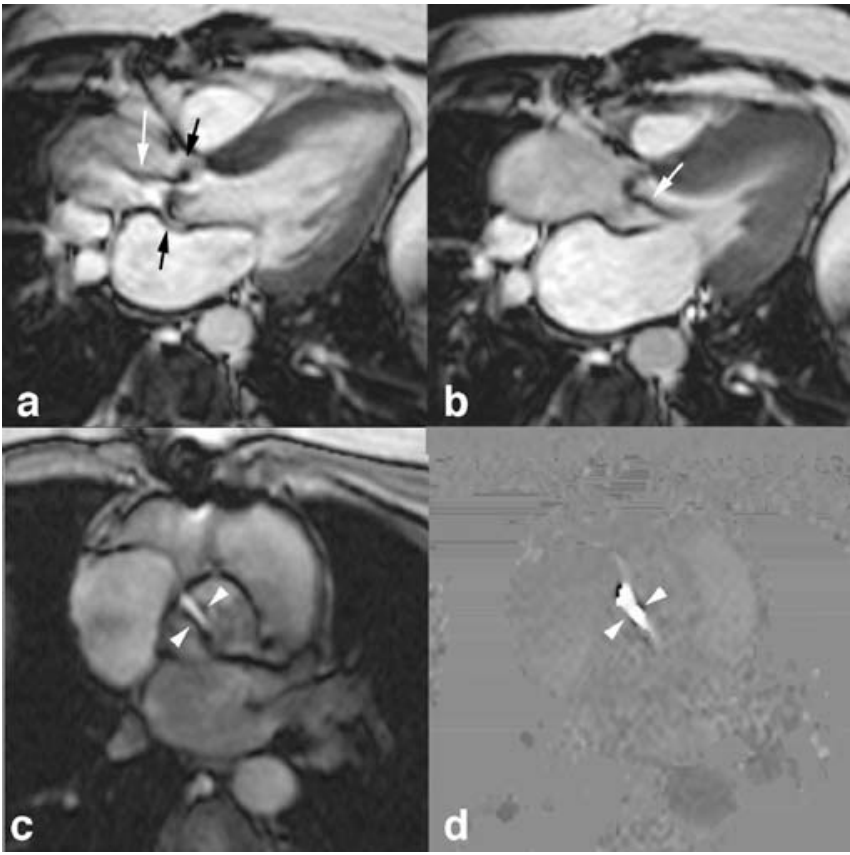

Fig. 8 Recurrence of severe aortic stenosis in a 38-year-old man. He had a history of rheumatic disease, with valvulotomy for aortic stenosis at age of 18. Balanced SSFP cine MRI of left ventricular inflow/outflow view during mid-systole (a) and early diastole (b) (movies 8A, 8B). Signal voids (white arrows) due to turbulent blood flow, shows concomitant presence of stenosis and regurgitation. Phase-contrast MRI, magnitude image (c) and phase image (d), reveals predominant stenotic defect (arrowheads) with peak systolic gradient of $170 \mathrm{mmHg}$ and planimetric aortic valve area of $0.7 \mathrm{~cm}^{2}$, and less severe aortic regurgitation $(22 \%)$. The aortic valve, is functionally bicuspid due to fusion of two aortic cusps which are extensively calcified (low signal intensity on leaflet tips; see movies). Note presence of dilated ascending aorta $(48 \mathrm{~mm})$

considered. This is paramount because aortic stenosis is mainly a disease of the elderly, in whom the risk of cardiac surgery may be particularly heightened by advanced age and other comorbidities. In the clinical practice, the transvalvular pressure gradients and functional aortic valve area (continuity equation) calculated by Doppler-TTE are often used to quantify aortic stenosis. However, these measurements may be misleading, as they depend on many factors, such as left ventricular pre- and afterload, left ventricle function and concomitant aortic regurgitation [39]. Moreover, the transaortic velocity calculated by continuouswave Doppler may be significantly underestimated if the angle between the ultrasound beam and the jet direction exceeds $20^{\circ}$ [40]. In clinical practice, this may occur quite frequently when a highly calcified and narrowed aortic valve induces eccentric jet flow. Moreover, by using the continuity equation, other potential sources of errors are related to left ventricle outflow tract area and velocity measurements. For these reasons, semi-invasive TEE planimetry of the aortic valve or even cardiac catheterization is often required. The former has shown to be quite accurate in calculating the anatomic aortic valve area; however, when extensive calcifications are present, TEE planimetry loses more of its accuracy [41]. For a long time cardiac catheterization has been regarded as the gold standard for quantification of aortic valve stenosis. However, it encompasses some relevant shortcomings. Cardiac catheterization is invasive, and moreover, catheter advancement through a calcified and stenotic aortic valve is associated with increased risk of cerebral embolism [42]. Secondly, measurement of the left ventricle-aorta pressure gradient by using the pullback maneuver may underestimate the severity of aortic stenosis in case of left ventricular dysfunction and when the pressure recovery phenomenon occurs. Moreover, when the Gorlin formula [43] is applied for measurement of aortic valve area, some inaccuracies may occur due to cardiac output calculation and the empirical variation of the Gorlin's constant [44].

The planimetry calculation of anatomic aortic valve area by using b-SSFP cine-MRI has been shown to be particularly helpful in overcoming most of these limitations. In contrast to GE imaging, the b-SSFP sequence is less dependent of flow velocity and flow turbulence, allowing more reliable contouring of stenotic area. In addition, the high signal-to-noise and contrast-to-noise ratios in conjunction with decreased susceptibility artifacts have rendered b-SSFP cine MRI a valid and reproducible tool for the aortic valve area measurement, even in the presence of important valve calcifications [45-47]. Moreover, the planimetric technique provides more accurate estimate of the severity of aortic valve stenosis than all other flowdependent methods. This is particularly useful in case of aortic stenosis and left ventricular dysfunction when flowdependent methods underestimate the aortic valve area [48]. In fact, transvalvular flow influences the pressure gradient measure but does not have any relevant consequences on the aortic valve anatomy calculated by planimetric analysis [49]. MRI is an excellent tool to

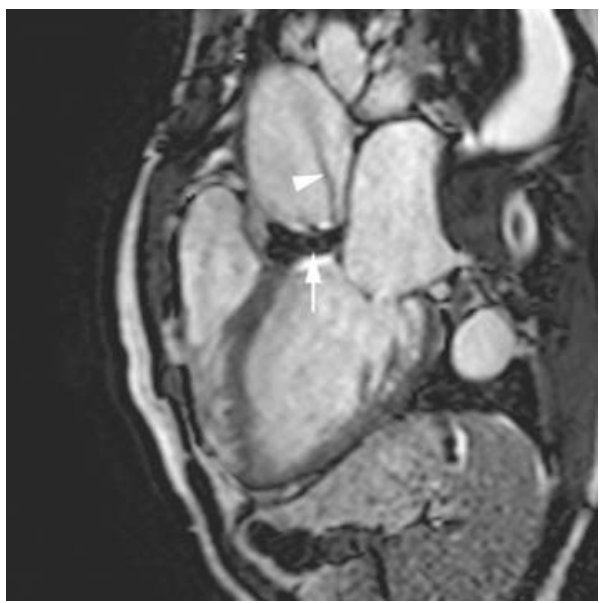

Fig. 9 Metallic aortic valve prosthesis in a 63-year-old man after Bentall surgery for ascending aortic dissection. Valve prothesis is visible as an area of signal void (white arrow) minimally disturbing image quality. Presence of periprosthetic leak (arrowhead) 
quantify the effects on increased afterload on left ventricular volumes, function and mass, as well as to monitor their changes after aortic valve replacement [50]. In patients with mixed aortic valve stenosis and regurgitation, we recommend to perform two or three phase-contrast MRI studies. The first measurement should be placed in the left ventricular outflow tract just beneath the aortic valve to quantify aortic regurgitation, the second one just above the aortic valve to quantify peak velocities, and optionally a third one perpendicular through the aortic valve to measure the aortic valve orifice (Fig. 8). The encoding velocity should careful adjusted to prevent aliasing but otherwise to maintain the highest flow sensitivity.

\section{Prosthetic valves}

At a magnetic field strength of 1.5 Tesla, all valvular prostheses can be safely imaged [51]. With the increased clinical use of high-field-strength magnets (i.e., 3.0-7.0 Tesla), safety concerns have again become relevant. In a recent paper, all artificial valves were considered safe in a static magnetic field up to 4.7 Tesla, except the CarpentierEdwards Physio Ring containing Elgiloy [52]. We recommend to consult the most current literature on MRI safety issues (http://www.MRIsafety.com). Most prosthetic valves are visible on bright-blood MRI as area of signal loss. The extent depends on the type of prosthesis and type of sequence used [53] (Fig. 9). Image quality and occurrence of artifacts are less hampered using the new balanced-
SSFP sequence. The latter often enables to evaluate valve prosthetic leaflets. However, the assessment of mechanical valve prosthesis dysfunction may be particularly intricate and small defects can be overlooked by cine MRI. For example, the presence of intra- or peri-prosthetic leakages is often hindered by the signal loss around the prosthesis. Phase-contrast imaging distally to the area of signal loss can be still accurately performed for flow and velocity measurements offering a reliable method for the noninvasive follow-up of patients after valvular surgery [54].

\section{Conclusion}

The use of cardiac MRI brings forth several strong arguments to become an important modality in the diagnosis and follow-up of VHD patients in the near future. Bright-blood cine MRI provides fast and reliable information on the presence of morphologic and functional valve abnormalities, and more importantly, MRI is likely the most accurate technique to quantify disease severity and impact on ventricular function. The very likely introduction of real-time MRI, 3D imaging techniques and slice tracking correction into clinical protocols in the near future will furthermore enhance its important role in the VHD field.

Acknowledgements P.G. Masci was supported by a grant from the European Society of Cardiology.

\section{References}

1. Simpson IA, Sahn DJ (1991) Quantification of valvular regurgitation by Doppler echocardiography. Circulation 84(3 Suppl):I188-I192

2. Segal J, Lerner DJ, Miller DC, Mitchell RS, Alderman EA, Popp RL (1987) When should Doppler-determined valve area be better than the Gorlin formula?: Variation in hydraulic constants in low flow states. J Am Coll Cardiol 9:1294-1305

3. Krombach GA, Kuhl H, Bucker A, Mahnken AH, Spuntrup E, Lipke C, Schroder J, Gunther RW (2004) Cine MR imaging of heart valve dysfunction with segmented true fast imaging with steady state free precession. J Magn Reson Imaging 19:59-67
4. Nikolaou K, Flohr T, Knez A, Rist C, Wintersperger B, Johnson T, Reiser MF, Becker CR (2004) Advances in cardiac CT imaging: 64-slice scanner. Int J Cardiovasc Imaging 20:535-540

5. Johnson TR, Nikolaou K, Wintersperger BJ, Leber AW, von Ziegler F, Rist C, Buhmann S, Knez A, Reiser MF, Becker CR (2006) Dual-source CT cardiac imaging: initial experience. Eur Radiol 16:1409-1415

6. Arai AE, Epstein FH, Bove KE, Wolff SD (1999) Visualization of aortic valve leaflets using black blood MRI. J Magn Reson Imaging 10:771-777

7. Schlosser T, Malyar N, Jochims M, Breuckmann F, Hunold P, Bruder O, Erber R, Barkhausen J (2007) Quantification of aortic valve stenosis in MRIcomparison of steady-state free precession and fast low-angle shot sequences. Eur Radiol 17:1284-1290

8. Evans AJ, Blinder RA, Herfkens RJ, Spritzer CE, Kuethe DO, Fram EK, Hedlund LW (1988) Effects of turbulence on signal intensity in gradient echo images. Invest Radiol 23:512-518
9. Wagner S, Auffermann W, Buser P, Lim TH, Kircher B, Pflugfelder P, Higgins CB (1989) Diagnostic accuracy and estimation of the severity of valvular regurgitation from the signal void on cine magnetic resonance images. Am Heart J 118:760-767

10. Suzuki J, Caputo GR, Kondo C, Higgins CB (1990) Cine MR imaging of valvular heart disease: display and imaging parameters affect the size of the signal void caused by valvular regurgitation. AJR Am J Roentgenol 155:723-727

11. Barkhausen J, Ruehm SG, Goyen M, Buck T, Laub G, Debatin JF (2001) MR evaluation of ventricular function: true fast imaging with steady-state precession versus fast low-angle shot cine MR imaging: feasibility study. Radiology 219:264-269 
12. Pruessmann KP, Weiger M, Scheidegger MB, Boesiger P (1999) SENSE: sensitivity encoding for fast MRI. Magn Reson Med 42:952-962

13. Lotz J, Meier C, Leppert A, Galanski M (2002) Cardiovascular flow measurement with phase-contrast MR imaging: basic facts and implementation. Radiographics 22:651-671

14. Bogren HG, Underwood SR, Firmin DN, Mohiaddin RH, Klipstein RH, Rees RS, Longmore DB (1987) Magnetic resonance velocity mapping: clinical application of a new technique. Br Heart J 57:404-412

15. Firmin DN, Nayler GL, Klipstein RH, Underwood SR, Rees RS, Longmore DB (1987) In vivo validation of MR velocity imaging. J Comput Assist Tomogr 11:751-756

16. Firmin DN, Nagler GL, Kilner PJ (1990) The application of phase shifts in NMR for flow measurements. Magn Reson Med 14:230-241

17. Kozerke S, Schwitter J, Pedersen EM, Boesiger P (2000) Aortic and mitral regurgitation: quantification using moving slice velocity mapping. J Magn Reson Imaging 14:106-112

18. Ichikawa Y, Sakuma H, Kitagawa K, Ishida N, Takeda K, Uemura S, Motoyasu M, Nakano T, Nozaki A (2003) Evaluation of left ventricular volumes and ejection fraction using fast steady-state cine MR imaging: comparison with left ventricular angiography. J Cardiovasc Magn Reson 5:333-342

19. Messroghli DR, Bainbridge GJ, Alfakih K, Jones TR, Plein S, Ridgway JP, Sivananthan MU (2005) Assessment of regional left ventricular function: accuracy and reproducibility of positioning standard short-axis sections in cardiac MR imaging. Radiology 235:229-236

20. Kaandorp TA, Lanb HJ, Bax JJ, van der Wall EE, de Ross A (2005) Magnetic resonance imaging of coronary arteries, the ischemic cascade, and myocardial infarction. Am Heart J 149:200-208

21. Mollet NR, Dymarkowski S, Volders W, Wathiong J, Herbots L, Rademakers FE, Bogaert J (2002) Visualization of ventricular thrombi with contrastenhanced magnetic resonance imaging in patients with ischemic heart disease. Circulation 106:2873-2876

22. Lascano ME, Schaad MS, Moodie DS, Murphy D Jr (2001) Difficulty in diagnosing double-chambered right ventricle in adults. Am J Cardiol 88:816-819
23. Chen CG, Thomas JD, Anconina J, Harrigan P, Mueller L, Picard MH, Levine RA, Weyman AE (1991) Impact of impinging wall jet on colour Doppler quantification of mitral regurgitation. Circulation 84:712-720

24. Enriquez-Sarano M, Avierinos JF, Messika-Zeitoun D, Detaint D, Capps M, Nkomo V, Scott C, Schaff HV, Tajik AJ (2005) Quantitative determinants of the outcome of asymptomatic mitral regurgitation. N Engl J Med 352:875-883

25. Zoghbi WA, Enriquez-Sarano M, Foster E, Grayburn PA, Kraft CD, Levine RA, Nihoyannopoulos P, Otto CM, Quinones MA, Rakowski H, Stewart WJ, Waggoner A, Weissman NJ; American Society of Echocardiography (2003) Recommendations for evaluation of the severity of native valvular regurgitation with two-dimensional and Doppler echocardiography. J Am Soc Echocardiogr 16:777-802

26. Sechtem U, Pflugfelder PW, Cassidy MM, White RD, Cheitlin MD, Schiller NB, Higgins CB (1988) Mitral or aortic regurgitation: quantification of regurgitant volumes with cine MR imaging. Radiology 167:425-430

27. Globits S, Frank H, Mayr H, Neuhold A, Glogar D (1990-1991) Quantification of regurgitant lesions by MRI. Int $\mathrm{J}$ Card Imaging 6:109-116

28. Kon MW, Myerson SG, Moat NE, Pennell DJ (2004) Quantification of regurgitant fraction in mitral regurgitation by cardiovascular magnetic resonance: comparison of techniques. J Heart Valve Dis 13:600-607

29. Fujita N, Chazouilleres AF, Hartiala JJ, O'Sullivan M, Heidenreich P, Kaplan JD, Sakuma H, Foster E, Caputo GR, Higgins CB (1994) Quantification of mitral regurgitation by velocity-encoded cine nuclear magnetic resonance imaging. J Am Coll Cardiol 23:951-958

30. Gelfand EV, Hughes S, Hauser TH, Yeon SB, Goepfert L, Kissinger KV, Rofsky NM, Manning WJ (2006) Severity of mitral and aortic regurgitation as assessed by cardiovascular magnetic resonance: optimizing correlation with Doppler echocardiography. J Cardiovasc Magn Reson 8:503-507

31. Grothues F, Smith GC, Moon JC, Bellenger NG, Collins P, Klein HU, Pennell DJ (2002) Comparison of interstudy reproducibility of cardiovascular magnetic resonance with twodimensional echocardiography in normal subjects and in patients with heart failure or left ventricular hypertrophy. Am J Cardiol 90:29-34
32. Bonow RO, Carabello B, de Leon AC, Edmunds LH Jr, Fedderly BJ, Freed MD, Gaasch WH, McKay CR, Nishimura RA, O'Gara PT, O'Rourke RA, Rahimtoola SH, Ritchie JL, Cheitlin MD, Eagle KA, Gardner TJ, Garson A Jr, Gibbons RJ, Russell RO, Ryan TJ, Smith SC Jr (1998) ACC/ AHA Guidelines for the management of patients with valvular heart disease. Executive Summary. A report of the American College of Cardiology/ American Heart Association Task Force on Practice Guidelines (Committee on Management of Patients With Valvular Heart Disease). J Heart Valve Dis 7:672-707

33. Murphy JG, Gersh BJ, Mair DD, Fuster V, McGoon MD, Ilstrup DM, McGoon DC, Kirklin JW, Danielson GK (1993) Long-term outcome in patients undergoing surgical repair of tetralogy of Fallot. N Engl J Med 329:593-599

34. Rebergen SA, Chin JG, Ottenkamp J, van der Wall EE, de Roos A (1993) Pulmonary regurgitation in the late postoperative follow-up of tetralogy of Fallot. Volumetric quantitation by nuclear magnetic resonance velocity mapping. Circulation 88(5 Pt 1):2257-2266

35. Carabello BA, Crawford FA Jr (1997) Valvular heart disease. N Engl J Med 337:32-41

36. Otto CM, Pearlman AS, Comess KA, Reamer RP, Janko CL, Huntsman LL (1986) Determinantion of stenotic aortic valve area in adults using Doppler echocardiography. J Am Coll Cardiol 7:509-517

37. Simpson IA, Maciel BC, Moises V, Shandas R, Elias W, Valdes-Cruz L, Hesselink JR, Chung KJ, Sahn DJ (1993) Cine magnetic resonance imaging and color Doppler flow mapping displays of flow velocity, spatial acceleration, and jet formation: a comparative in vitro study. Am Heart J 126:1165-1174

38. Selzer A (1987) Changing aspects of the natural history of valvular aortic stenosis. N Engl J Med 317:91-98

39. Danielsen R, Nordrehaug JE, Vik-Mo H (1989) Factors affecting Doppler echocardiographic valve area assessment in aortic stenosis. Am J Cardiol 63:1107-1111

40. Rahimtoola SH, Frye RL (2000) Valvular heart disease. Circulation 102: IV24-IV33 
41. Bernard Y, Meneveau N, Vuillemenot A, Magnin D, Anguenot T, Schiele F, Bassand JP (1997) Planimetry of aortic valve area using multiplane transoesophageal echocardiography is not a reliable method for assessing severity of aortic stenosis. Heart 78:68-73

42. Omran H, Schmidt H, Hackenbroch M, Illien S, Bernhardt P, von der Recke G, Fimmers R, Flacke S, Layer G, Pohl C, Luderitz B, Schild H, Sommer T (2003) Silent and apparent cerebral embolism after retrograde catheterisation of the aortic valve in valvular stenosis: a prospective, randomised study. Lancet 361:1241-1246

43. Gorlin R (1951) Hydraulic formula for calculation of the area of the stenotic mitral valve, other cardiac valve and central circulatory shunts. Am Heart J 41:1-18

44. Cannon SR, Richards KL, Crawford MH, Folland ED, Pierpont G, Sethi GK, Hammermeister KE (1988) Inadequacy of the Gorlin formula for predicting prosthetic valve area. Am J Cardiol 62:113-116
45. Kupfahl C, Honold M, Meinhardt G, Vogelsberg H, Wagner A, Mahrholdt H, Sechtem U (2004) Evaluation of aortic stenosis by cardiovascular magnetic resonance imaging: comparison with established routine clinical techniques. Heart 90:893-901

46. Reant P, Lederlin M, Lafitte S, Serri K, Montaudon M, Corneloup O, Roudaut R, Laurent F (2006) Absolute assessment of aortic valve stenosis by planimetry using cardiovascular magnetic resonance imaging: comparison with transesophageal echocardiography, transthoracic echocardiography, and cardiac catheterisation. Eur J Radiol 59:276-283

47. John AS, Dill T, Brandt RR, Rau M, Ricken W, Bachmann G, Hamm CW (2003) Magnetic resonance to assess the aortic valve area in aortic stenosis: how does it compare to current diagnostic standards? J Am Coll Cardiol 42:519-526

48. Carabello BA (2004) Is it very too late to operate on the patient with valvular heart disease? J Am Coll Cardiol 44:376-383

49. Tardif JC, Rodrigues AG, Hardy JF, Leclerc Y, Petitclerc R, Mongrain R, Mercier LA (1997) Simultaneous determination of aortic valve area by the Gorlin formula and by transesophageal echocardiography under different transvalvular flow conditions. Evidence that anatomic aortic valve area does not change with variations in flow in aortic stenosis. J Am Coll Cardiol 29:12961302
50. Lamb HJ, Beyerbracht HP, de Roos A, van der Laarse A, Vliegen HW, Leujes F, Bax JJ, Van der Wall EE (2002) Left ventricular remodeling early after aortic valve replacement: differential effects on diastolic function in aortic valve stenosis and aortic regurgitation. J Am Coll Cardiol 40:2182-2188

51. Shellock FG, Crues JV (2004) MR procedures: biologic effects, safety, and patient care. Radiology 232:635-652

52. Edwards MB, Ordidge RJ, Hand JW, Taylor KM, Young IR (2005) Assessment of magnetic field (4.7 T) induced forces on prosthetic heart valves and annuloplasty rings. J Magn Reson Imaging 22:311-317

53. Deutsch HJ, Bachmann R, Sechtem U, Curtius JM, Jungehulsing M, Schicha H, Hilger HH (1992) Regurgitant flow in cardiac valve prostheses: diagnostic value of gradient echo nuclear magnetic resonance imaging in reference to transesophageal two-dimensional color Doppler echocardiography. J Am Coll Cardiol 19:1500-1507

54. Fontaine AA, Heinrich RS, Walker PG, Pedersen EM, Scheidegger MB, Boesiger P, Walton SP, Yoganathan AP (1996) Comparison of magnetic resonance imaging and Laser Doppler Anemometry velocity measurements downstream of replacement heart valves: implications for in vivo assessment of prosthetic valve function. $J$ Heart Valve Dis 5:66-73 